\title{
Astrometry of red supergiant VY Canis Majoris with VERA
}

\author{
Y. K. Choi ${ }^{1,2}$, T. Hirota ${ }^{2}$, M. Honma ${ }^{2}$, \\ and H. Kobayashi ${ }^{2}$ \\ ${ }^{1}$ Department of Astronomy, Graduate School of Science, The University of Tokyo, \\ 7-3-1 Hongo, Bunkyo-ku, Tokyo 113-0033, Japan \\ ${ }^{2}$ Mizusawa VERA Observatory, National Astronomical Observatory of Japan, \\ 2-21-1 Osawa, Mitaka, Tokyo 181-8588, Japan \\ email: yoonkyung.choi@nao.ac.jp
}

\begin{abstract}
We present observational results on the red supergiant VY Canis Majoris with VERA. We have observed $22 \mathrm{GHz} \mathrm{H}_{2} \mathrm{O}$ masers and $43 \mathrm{GHz} \mathrm{SiO}$ masers (v=1 and $2 \mathrm{~J}=1-0$ ) around VY CMa for 13 months. We succesfully detected a parallax of $0.87 \pm 0.08$ mas, corresponding to the distance of $1.15_{-0.09}^{+0.10} \mathrm{kpc}$ using $\mathrm{H}_{2} \mathrm{O}$ masers. As the result of phase-referencing analyses, we have measured absolute positions for both $\mathrm{H}_{2} \mathrm{O}$ masers and $\mathrm{SiO}$ masers. The $\mathrm{H}_{2} \mathrm{O}$ maser features show rapid expansion off the central star.
\end{abstract}

Keywords. astrometry, masers, stars: distances, supergiants, stars: individual (VY CMa)

\section{Introduction}

VY CMa is one of the most luminous red supergiants in our galaxy. Its luminosity is $4.3 \times 10^{5} \mathrm{~L}_{\odot}$ (Humphreys, Helton \& Jones 2007) and distance is estimated to be 1.5 kpc (Lada \& Reid 1978). VY CMa is ejecting large amount of gas and dust at a high mass loss rate of $4 \times 10^{-4} \mathrm{M}_{\odot} \mathrm{yr}^{-1}$ (Danchi et al. 1994). $\mathrm{H}_{2} \mathrm{O}$ and $\mathrm{SiO}$ masers are commonly found in circumstellar envelopes (CSE) of supergiants. They are important tools to study structures and kinematics of the CSE, the mass-loss process and evolution of massive stars.

\section{Parallax measurements of VY CMa}

We have observed $\mathrm{H}_{2} \mathrm{O}$ masers around the red supergiant VY CMa at 10 epochs with VERA for 13 months. The source J0725-2640, whose angular distanc from VY CMa is $1.059 \mathrm{deg}$, was observed as a positional reference source. Using a bright $\mathrm{H}_{2} \mathrm{O}$ maser spot at the LSR velocity of $0.55 \mathrm{~km} \mathrm{~s}^{-1}$, we succesfully detected a trigonometric parallax of $0.87 \pm 0.08$ mas, corresponding to the distance of $1.15_{-0.09}^{+0.10} \mathrm{kpc}$. Figure 1 shows the results of our positional measurements for this $\mathrm{H}_{2} \mathrm{O}$ maser spot. The astrometric accuracy in right ascension is better than that in declination. A possible reason for this disparity could be uncertainty in the atmospheric zenith delay.

\section{3. $\mathrm{H}_{2} \mathrm{O}$ masers and $\mathrm{SiO}$ masers around VY CMa}

$\mathrm{SiO}$ masers are detected in a spherical shell at $2-4 \mathrm{R}_{*}$ from the central star (Diamond et al. 1994) but the $\mathrm{H}_{2} \mathrm{O}$ masers are distributed over a wider region than $\mathrm{SiO}$ masers. The resulting phase-referencing analysis indicates that the absolute positions for both $\mathrm{H}_{2} \mathrm{O}$ masers and $\mathrm{SiO}$ masers are measured with an accuracy better than 1 mas. Figure 2 

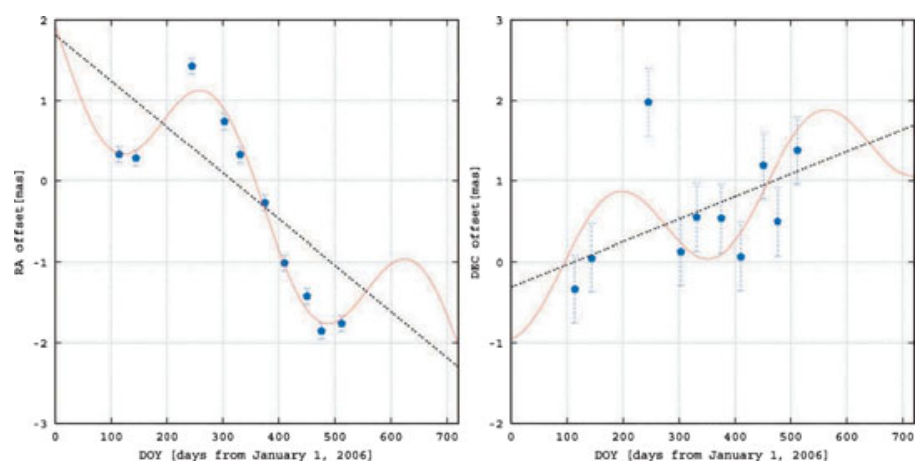

Figure 1. Results of position measurements for $\mathrm{H}_{2} \mathrm{O}$ maser spot at the LSR velocity of $0.55 \mathrm{~km}$ $\mathrm{s}^{-1}$ in VY CMa. Left panel: The motion of the maser spot in RA as a function of time over 13 months. Right panel: The same as left panel but for Dec. Solid lines represent the best fit model for annual parallax and proper motion of a maser spot. Dotted lines represent proper motion

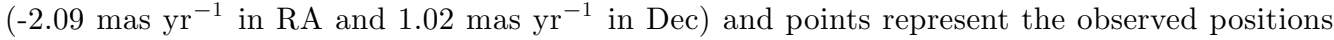
of a maser spot with error bars indicating the standard deviation from least-squares analysis (0.10 mas in RA and 0.42 mas in Dec).

shows the distribution of $\mathrm{H}_{2} \mathrm{O}$ and $\mathrm{SiO}$ maser features. From the position of $\mathrm{SiO}$ maser ring, we can estimate the position of stellar component. The $\mathrm{H}_{2} \mathrm{O}$ maser features move away from the central star with the velocity of $5-30 \mathrm{~km} \mathrm{~s}^{-1}$. The diameter of $\mathrm{SiO}$ maser feature is about 40 mas, corresponding to $46 \mathrm{AU}$ at the distance of $1.15 \mathrm{kpc}$. If the $\mathrm{SiO}$ masers are formed at 2 stellar radii, then the radius of VY CMa should be about $1200 \mathrm{R}_{\odot}$.

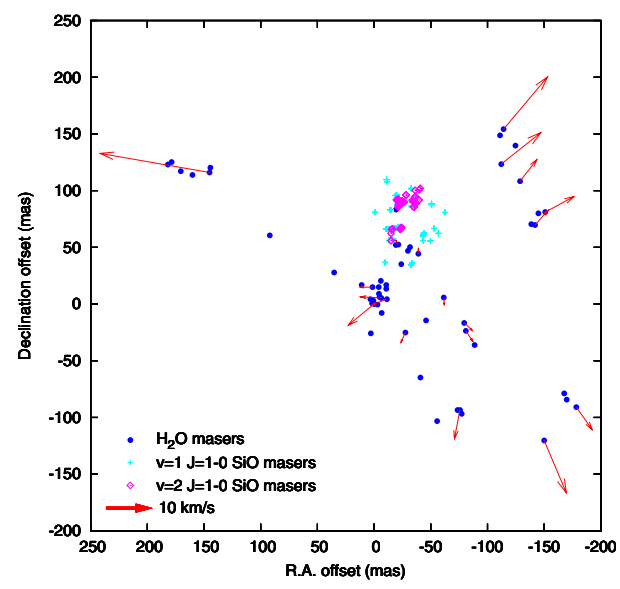

Figure 2. Direct Comparison of absolute positions between $\mathrm{H}_{2} \mathrm{O}$ masers and $\mathrm{SiO}$ masers are possible with phase-referencing analyses. Closed circle, cross and open diamond represent $\mathrm{H}_{2} \mathrm{O}$ masers, $\mathrm{v}=1$ and $\mathrm{v}=2 \mathrm{~J}=1-0 \mathrm{SiO}$ masers, respectively. Arrows represent the proper motion of $\mathrm{H}_{2} \mathrm{O}$ masers from which the mean motion has been subtracted.

\section{References}

Danchi, W. C., Bester, M. Degiacomi, C. G., Greenhill, L. J., \& Townes, C. H. 1994, AJ, 107, 1469.

Diamond, P. J., Kemball, A. J., Junor, W., et al. 1994, ApJ, 430, L61.

Humphreys, R. M., Helton, L. A. \& Jones, T. J. 2007, AJ, 133, 2716.

Lada, C. J. \& Reid, M. J. 1978, ApJ, 219, 95. 